\title{
Lamotrigine induced seizures in juvenile myoclonic epilepsy
}

\author{
Betül Acar, Ersel Dağ*, Yakup Türkel \\ Department of Neurology, Faculty of Medicine, Kırlkkale University, Kırıkale, Turkey
}

\section{ARTICLE INFO ABSTRACT}

\section{Article History}

Received $\quad 15 / 04 / 2013$

Accepted $\quad 10 / 05 / 2013$

\section{* Correspondence to:}

Ersel Dă̆

Department of Neurology,

Kırıkkale University, Kırıkkale, Turkey

e-mail: erseldag@yahoo.com

\section{Keywords:}

Antiepileptic drug

Seizure

Juvenile myoclonic epilepsy

Lamotrigine

\begin{abstract}
Juvenile myoclonic epilepsy (JME) is a common epileptic syndrome. Before the development of the new antiepileptic drugs (AEDs) after the 1990s, Valproate (VPA) was the first-line treatment in juvenile myoclonic epilepsy. However, the frequent adverse effects and the risk for teratogenicity have resulted in a search for alternative therapies especially in women. Lamotrigine (LTG), topiramate, and levetiracetam, have been used as monotherapy or adjunctive therapy for juvenile myoclonic epilepsy in small patient series. We report a case of a thirty one-years-old female suffered from severe aggravation in myoclonic and generalized tonic-clonic (GTC) seizures after decreasing the lose of VPA and initiating LTG.
\end{abstract}

J. Exp. Clin. Med., 2013; 30:207-208

This case report was presented as a poster in the 7th World Congress on Controversies in Neurology (CONy), April 11-14, 2013.

\section{Introduction}

Juvenile myoclonic epilepsy (JME) is a common epileptic syndrome accounts for 5 to $11 \%$ of all cases of epilepsy. Although it has quite typical clinical presentation and electroencephalographic (EEG) findings, patients of JME have often misdiagnosed and/or have delayed diagnosis (Atakli et al., 1998).

Valproate (VPA) is still the first choice antiepileptic drug in the treatment of primary JME. However, the use of VPA particularly in fertile age groups increases the risk of fetal malformation and may delay the neuronal development of the fetus (Duncan, 2007). Therefore, the usage of new generation antiepileptic drugs such as lamotrigine (LTG) levetiracetam, topiramate have been advocated especially in young women (Auvin, 2008). However, LTG may increase the frequency of myoclonic and GTC seizures (Mantoan and Walker, 2011).

In this case report, it has been presented that 31-yearsold female who was started VPA with the diagnosis of idiopathic generalized epilepsy and who experienced severe aggravation in myoclonic and generalized tonic-clonic seizures when LTG $(50 \mathrm{mg} / \mathrm{d})$ was prescribed in association to VPA $(500 \mathrm{mg} / \mathrm{d})$ after reduction of VPA therapy because of the side effects.

\section{Case}

Thirty-one-years-old female patient was taking $1000 \mathrm{mg} /$ day VPA therapy with a diagnosis of idiopathic generalized epilepsy for more than ten years. The patient was admitted to a neurologist due to convulsions re-occurring. She had complaints of weight gain and hair loss. As she was in the period of fertility, the possible teratogenic side effects of VPA was also taken into account, VPA dose was reduced to $500 \mathrm{mg} /$ day and LTG $50 \mathrm{mg}$ / day was initiated.

During follow-up, under combination therapy the patient suffered from frequent convulsions which was required to visit the emergency department due to convulsions almost every day. She admitted our clinic with these complaints. Twenty minutes after the admission to inpatient clinic, a GTC seizure was observed which was lasting about two minutes and followed by postictal confusion. Routine laboratory examinations were normal and postictal $2^{\text {nd }}$ hour EEG revealed generalized spikes and multiple spike-slow wave paroxysms indicating JME (Fig. 1).

Due to increased complaints, the combination therapy was stopped and dose of VPA was increased gradually to $1500 \mathrm{mg}$ / day after informing the patient and her husband about the potential side effects and teratogenicity. After treat- 


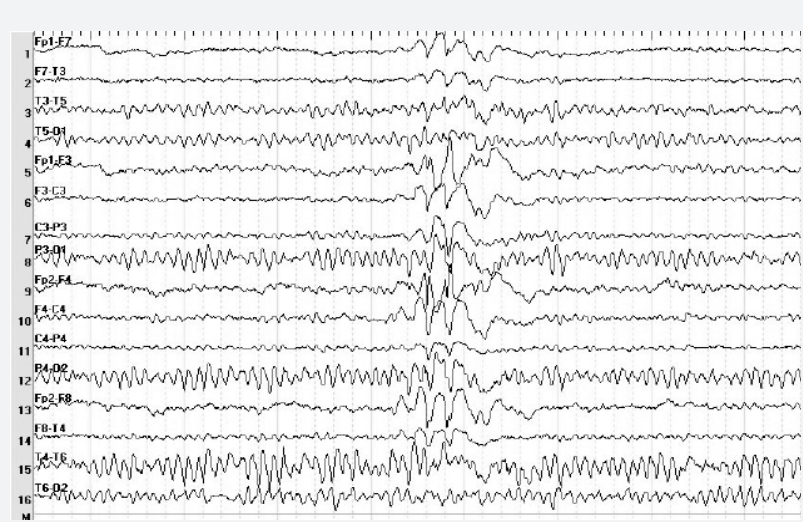

Fig. 1. Generalized spikes, multiple spike-slow wave paroxysms

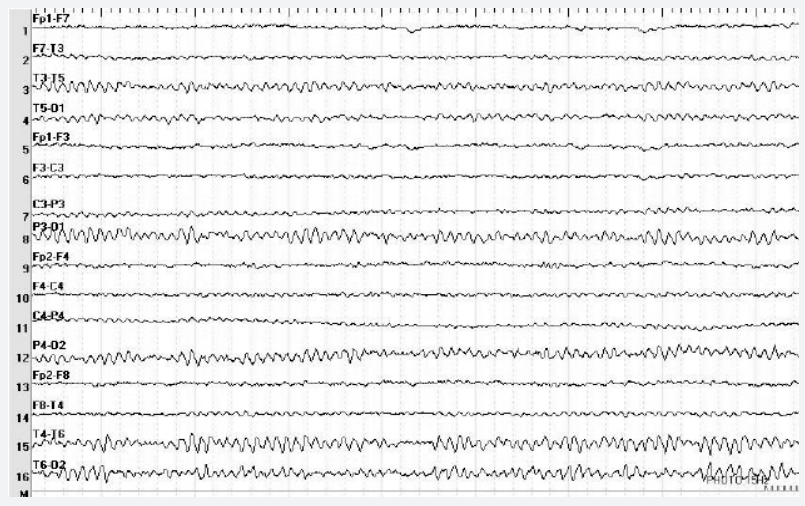

Fig. 2. Normal EEG

ment with high dose VPA, she has been doing well without any seizures for 1 year follow-up. Under the treatment of high dose VPA theraphy awake EEG was normal (Fig. 2).

\section{Discussion}

Although there are typical clinical and EEG findings exists for JME, patients are quite often misdiagnosed and appropriate treatment is delayed. Many patients do not mention about myoclonic jerks as they usually do not found as significant
(Mehndiratta and Aggarwal, 2002). Our case also did not mentioned from myoclonic jerks, before admitted to our inpatient clinic. However, GTC and absence type seizures may accompany in 80 to $97 \%$ patients of JME (Mehndiratta and Aggarwal, 2002). We did not have knowledge about the previous EEGs of our patient.

Generalized spikes and multiple spike slow wave patterns were observed in short-term EEG recordings taken during the observation period in our clinic.

VPA is the first choice in the treatment of JME. If VPA therapy is ineffective or the patient is in the period of fertility, drugs such as LTG, levetiracetam and topiramate may be preferred (Mantoan and Walker, 2011). However, It have also been reported that LTG may increase the GTC and myoclonic seizures. LTG may be the cause of increased frequencies of GTC and myoclonic seizures in this case, in addition reduction of VPA may be another reason. Considering these probabilities we had decided to stop LTG with tapering the dose while simultaneously increasing VPA dose. During this regimen the seizures had disapperaed and no increased side effects due to VPA was observed.

Bodenstein-Sachar et al.(2011) reported that respond to treatment with LTG is well in patients with JME without idiopathic generalized epilepsy.

Janszky et al. (2000) have reported that LTG was associated with increased rate in myoclonic jerks with increasing serum levels, both in LTG monotherapy and in the combination therapy with VPA, in patients with refractory idiopathic generalized epilepsy. Similarly our patient was also suffered from increased frequency of seizures when the combination of VPA and LTG was used.

Crespel et al. (2005) have observed that myoclonic jerks have emerged during treatment with LTG in the five patients with idiopathic generalized epilepsy. The seizures was stopped when the doses were decreased about $25-50 \%$ in three of the patients however for the remaining two patients, LTG therapy had to be stopped for cessation of the seizures. In conclusion, clinicians should be aware that LTG may cause an increase in seizures in patients with JME when occurred together with GTC.

\section{REFERENCES}

Atakli, D., Sözüer, D., Atay, T., Baybas, S., Arpaci, B., 1998. Misdiagnosis and treatment in juvenile myoclonic epilepsy. Seizure 7, 63-66.

Auvin, S., 2008. Treatment of juvenile myoclonic epilepsy. CNS Neurosci. Ther. 14, 227-233.

Bodenstein-Sachar, H., Gandelman-Marton, R., Ben-Zeev, B., Chapman, J., Blatt, I., 2011. Outcome of LTG treatment in juvenile myoclonic epilepsy. Acta Neurol. Scand. 124, 22-27.

Crespel, A., Genton, P., Berramdane, M., Coubes, P., Monicard, C., Baldy-Moulinier M., Gelisse, P., 2005. LTG associated with exacerbation or de novo myoclonus in idiopathic generalized epilepsies. Neurology. 65, 762-764.

Duncan, S., 2007. Teratogenesis of sodium VPA. Curr. Opin. Neurol. 20, 175-180.

Janszky, J., Rásonyi, G., Halász, P., Olajos, S., Perenyi, J., 2000. Disabling erratic myoclonus during lamotrigine therapy with high serum level-report of two cases. Clin. Neuropharmacol. 23, 86-89.

Mantoan, L., Walker, M., 2011. Treatment options in juvenile myoclonic epilepsy. Curr. Treat. Options Neurol. 13, 355-370.

Mehndiratta, M.M., Aggarwal, P., 2002. Clinical expression and EEG features of patients with juvenile myoclonic epilepsy (JME) from North India. Seizure. 11, 431-436. 